\section{MRI Yield of Posterior Fossa Strokes in 'Dizzy' Patients}

\author{
Anthea Ki Kwan Lam* and John Brunton \\ Royal Derby Hospital, Uttoxeter New Road, Derby, UK
}

\begin{abstract}
Objectives: To review the sensitivity of symptoms of dizziness in diagnosing Posterior Fossa Strokes. To assess the appropriateness of MRI scan in this subtype of patients

Methods: 588 patients under went MRI within a one-year period. Data was ascertained through requests and results on ICM computer results/investigation system at Royal Derby Hospital to determine symptoms at presentation and the presence or absence of an acute infarct.

Results: Of the 588 patients who had symptoms of dizziness and vertigo, 24 patients $(4.08 \%)$ had an acute posterior Fossa infarct on diffusion weighted imaging on MRI, meaning that $95.92 \%$ did not and of these, only a small minority had other pathology such as a mass lesion or acoustic neuroma. Only one patient had bilateral posterior circulation infarcts. $3.90 \%$ of those with negative MRI scans had a stroke but in the anterior circulation. $67.86 \%$ patients undergoing MRI scans for their symptoms had reports of no abnormal findings and no cause for their vertigo and dizziness.

Conclusion: Clinical examination findings should be used to determine appropriateness of MRI scans rather than relying purely on patients' reported symptoms of dizziness and vertigo which can be subjective and non-specific. MRI also has such a high negative predictive value with $67.86 \%$ of any pathology and $95.92 \%$ negative predictive value for presence of posterior Fossa infarct. Had case notes been available, it would be interesting to see the number of patients who had a full neurological examination prior to request for MRI.

Advances in knowledge: Increasingly, MRI is requested for patients presenting with dizziness. Results show this alone is not a sensitive predictor of Posterior Fossa Stroke. Thorough history, neurological examinations and simple procedures such as Dix-Hallpike, a manoeuvre where the patient's head is tilted 45 degrees before being swiftly lowering the patient and looking for signs of nystagmus, can help distinguish peripheral/central aetiology.
\end{abstract}

\section{Background}

Dizziness is a common presenting complaint amongst patients especially given the vagueness of this term to encompass vertigo,

*Corresponding author: Anthea Ki Kwan Lam, Royal Derby Hospital, Uttoxeter New Road, Derby, DE22 3NE, UK, Tel: +44 01322340131; Fax: +44 01332785566; E-mail: Anthea.lam@nhs.net

Citation: Lam AKK, Brunton J (2015) MRI Yield of Posterior Fossa Strokes in 'Dizzy' Patients. J Nucl Med Radiol Radiat Ther 1: 002.

Received: November 13, 2015; Accepted: February 12, 2016; Published: February 27, 2016 pre-syncope and unsteadiness [1]. The posterior circulation is defined primarily by the vertebro-basilar arterial system, which arises from the subclavian arteries. Infarcts affecting the superior or Posterior Inferior Cerebellar Arteries (PICA) can cause symptoms of vertigo.

Many patients with unexplained dizziness ultimately undergo an MRI scan to rule out a posterior circulation ischaemic stroke. Prior research showed that only $3.2 \%$ of patients presenting with dizziness, vertigo or imbalance were diagnosed with a stroke or Transient Ischaemic Attack (TIA) [2]. Vestibular symptoms such as vertigo can be attributed to both peripheral causes such as vestibular neuritis or central causes like brainstem/cerebellar stroke. In a study of 240 patients who presented with vertigo lasting more than a day and no other focal neurological deficits, $10 \%$ were diagnosed with cerebellar strokes but ischaemia associated with vertigo is often accompanied by other brainstem signs [3]. However, only $17 \%$ of patients with confirmed PICA strokes had symptoms of vestibular neuritis [3]. Manoeuvres such as Dix-Hallpike can help distinguish between peripheral or central aetiology since none of the patients who had symptoms of vestibular neuritis due to ischaemic stroke had a positive head thrust [4]. Nevertheless, it has been argued that MRI plays an important role in differentiating between peripheral and central aetiology when clinical diagnosis fails [5].

CT scan has been proven to have poor sensitivity in diagnosing posterior Fossa strokes in patients with dizziness and vertigo as it provides suboptimal visualisation of structures secondary to artefact produced by bony structures of the cranial base and therefore early ischaemic events are missed $[5,6]$. Accordingly, diffusion weighted MRI scans is more commonly used, but even this modality misses up to $20 \%$ posterior Fossa infarcts in the first $24-48$ hours of presentation [1]. The yield of MRI for stroke in this cohort of patients was only $21 \%$, with a diagnostic impact on management for $22 \%$ of these patients [5]. This unreliability of MRI scan is reinforced by a study which showed that diffusion weighted imaging was falsely negative in 12 of patients with acute vestibular symptoms and posterior Fossa strokes [7].

\section{Method}

\begin{tabular}{|c|c|}
\hline Sample Population & $\begin{array}{l}\text { All patients presenting with vestibular symptoms undergo- } \\
\text { ing MRI scans between the period 01-09-2014 and 31-08- } \\
2015 .\end{array}$ \\
\hline Audit Methodology & Retrospective \\
\hline Sample Size & 588 \\
\hline
\end{tabular}

A total of 603 MRI scans were performed for patients presenting with vertigo and dizziness with suspicion of cerebellar or brainstem infarcts during the one year period analysed at Royal Derby Hospital. Prior research showed that there is a low yield of posterior circulation infarcts diagnosed on MRI in this cohort of patients with dizziness $[1,5]$.

Data was accumulated for those 603 patients with MRI scans through screening of patients who underwent MRI scan in the last year and key words on the request or report cards included 'cerebellar infarct', 'cerebellar stroke, 'brainstem stroke, 'vertigo', 'dizziness', 'ataxia' or 'unsteadiness'. 
15 patients were removed from the data analysis for the following reasons:

- MRI heart scan - to assess for cardiomyopathy post partum (1)

- MRI face - parotid mass with incidental finding of old occipital infarct (1)

- No dizziness/vertigo but incidental finding of posterior circulation infarct (3)

- Asymptomatic and negative MRI scan (10)

Clinical indication for MRI scan was ascertained through history noted on the ICM (results and investigation ordering system at Royal Derby Hospital) requests and formal reporting of findings recorded. The remaining 588 patients in the sample were divided according to whether scans reported presence of posterior circulation infarct. Patients who had previous diagnosis of posterior Fossa strokes and had no new findings in comparison to old imaging and those who had anterior circulation infarcts were not included as positive findings.

Data selected from the 588 patients included presence or absence of stroke, the area of infarct and the presenting symptoms. All scans performed were performed within 24 hours of presentation and therefore were acute strokes. Patients who presented with vertigo or dizziness were isolated and these were correlated with those who had strokes.

\section{Results}

\begin{tabular}{|c|c|c|}
\hline $\begin{array}{c}\text { Patients undergoing MRI for vestibular } \\
\text { symptoms }\end{array}$ & Number & Percentage \% \\
\hline Presence of Posterior Fossa Infarct & $24 / 588$ & 4.1 \\
\hline No evidence of Posterior Fossa Infarct & $564 / 588$ & 95.9 \\
\hline Anterior circulation infarct - new diagnosis & $22 / 588$ & 3.7 \\
\hline No acute findings noted on MRI report & $399 / 588$ & 67.9 \\
\hline
\end{tabular}

Of the 588 patients who had symptoms of dizziness and vertigo, 24 patients (4.08\%) had posterior Fossa infarct on diffusion weighted imaging on MRI, meaning that $95.92 \%$ did not and of these, only a small minority had other pathology such as a mass lesion or acoustic neuroma. Only one patient had bilateral posterior circulation infarct. $3.90 \%$ of those with negative MRI scans had a stroke but in the anterior circulation. $67.86 \%$ patients undergoing MRI scans for their symptoms had reports of no abnormal findings and no cause for their vertigo and dizziness.

\section{Conclusion}

Analysis of the data showed that dizziness and vertigo is not a sensitive predictive factor for diagnosis of posterior Fossa infarct. We can therefore conclude that dizziness is a vague symptom; in fact, many of the scans performed showed normal brain imaging and normal internal acoustic meatus. Only 3.90\% of those without posterior Fossa infarcts had an infarct elsewhere in the brain. Most of the other patients (67.86) had normal imaging with no cause found for their symptoms. There was only $0.34 \%$ difference between diagnoses of new onset posterior and anterior circulation strokes underlying the fact that 'posterior circulation symptoms' of vertigo, ataxia, dizziness and vomiting are non-specific and cannot predict the aetiology.

Findings from this small study suggest that similar to Kabra's research 'Diagnostic yield and impact of MRI for acute ischaemic stroke in patients presenting with dizziness and vertigo', Dix-Hallpike manoeuvres should be performed to distinguish peripheral and central aetiology since it is a good predictive value of posterior Fossa stroke. As the manoeuvre is such a simple bedside test, it should perhaps be used more in clinical practice to differentiate between aetiology rather than relying solely on 'diagnostic' scans [4]. In conclusion, this study suggests that clinical examination findings should be used to determine appropriateness of MRI scans rather than relying purely on patients' reported symptoms of dizziness and vertigo. This is because such reported symptoms are often subjective and non-specific, and they also have a high negative predictive value with $67.86 \%$ of any pathology and $95.92 \%$ negative predictive value for presence of posterior Fossa infarct. Had case notes been available, it would be interesting to see the number of patients who had a full neurological examination prior to request for MRI.

Kabra et al., came to an alternative conclusion where the yield was $21 \%$ with diagnostic impact of MRI on management being 22\% [4] meaning clinicians should have a low threshold for requesting MRI if patients exhibit these features. However, this research study would suggest that vestibular symptoms are non-specific for diagnosing posterior Fossa infarct given the low percentage of positive results amongst the large cohort in this study. The majority of MRI are normal (67\%) and only $8 \%$ (anterior and posterior) infarct demonstrate stroke. In conclusion, this study suggests that clinical examination should take precedence over relying on MRI scans in patients with dizziness for a diagnosis.

\section{References}

1. Tarnutzer AA, Berkowitz AL, Robinson KA, Hsieh YH, Newman-Toker DE (2011) Does my dizzy patient have a stroke? A systematic review of bedside diagnosis in acute vestibular syndrome. CMAJ 183: 571-592.

2. Nouh A, Remke R, Ruland S (2014) Ischaemic posterior circulation stroke: a review of anatomy, clinical presentations, diagnosis and current management. Front Neurol 5: 30.

3. Lee H, Sohn SI, Cho YW, Lee SR, Ahn BH, et al. (2006) Cerebellar infarction presenting isolated vertigo: frequency and vascular topographical patterns. Neurology 67: 1178-1183.

4. Kabra R, Robbie H, Connor SE (2015) Diagnostic yield and impact of MRI for acute ischaemic stroke in patients presenting with dizziness and vertigo. Clin Radiol 70: 736-742.

5. Kerber KA, Brown DL, Lisabeth LD, Smith MA, Morgenstern LB (2006) Stroke among patients with dizziness, vertigo, and imbalance in the emergency department: a population-based study. Stroke 37: 2484-2487.

6. Schneider JI, Olshaker JS (2012) Vertigo, vertebrobasilar disease, and posterior circulation ischemic stroke. Emerg Med Clin North Am 30: 681-693.

7. Kattah JC, Talkad AV, Wang DZ, Hsieh YH, Newman-Toker DE (2009) HINTS to diagnose stroke in the acute vestibular syndrome: three-step bedside oculomotor examination more sensitive than early MRI diffusion-weighted imaging. Stroke 40: 3504-3510. 